\title{
Singularly perturbed integro-differential equations with rapidly oscillating coefficients and with rapidly changing kernel in the case of a multiple spectrum
}

\author{
BURKHAN KALIMBETOV \\ Ahmet Yassawi University \\ Natural Sciences Institute \\ Sattarkhanov ave. 29 \\ 161200, Turkestan \\ KAZAKHSTAN
}

\author{
VALERY SAFONOV \\ National Research University \\ Moscow Power Engineering Institute \\ Krasnokazarmennaya Street 14, \\ 111250, Moscow \\ RUSSIA
}

\begin{abstract}
The paper investigates a system with rapidly oscillating coefficients and with a rapidly decreasing kernel of the integral operator. Previously, only differential problems of this type were studied in which the integral term was absent. The presence of an integral operator significantly affects the development of an algorithm for asymptotic solutions, for the implementation of which it is necessary to take into account essentially singularities generated by the rapidly decreasing spectral value of the kernel of the integral operator. In addition, resonances can arise in the problem under consideration (i.e., the case can be realized when an integer linear combination of the eigenvalues of the rapidly oscillating coefficient coincides with the points of the spectrum of the limiting operator over the entire considered time interval), as well as the case where the eigenvalue of the rapidly oscillating coefficient coincides with the points spectrum of the limiting operator. This case generates a multiple spectrum of the original singularly perturbed integro-differential system. A similar problem was previously considered in the case of a simple spectrum. More complex cases of resonance (for example, point resonance) require more careful analysis and are not considered in this article.
\end{abstract}

Key-Words:- singular perturbation; integro-differential equation; rapidly oscillating coefficient; regularization; asymptotic convergence.

Received: December 2, 2020. Revised: March 9, 2021. Accepted: March 22, 2021. Published: April 8, 2021.

\section{Introduction}

Various applied problems related to dynamic stability and the properties of media with a periodic structure lead to differential equations with rapidly oscillating coefficients. The presence of high-frequency terms in them prevents an efficient numerical calculation of approximate solutions. Therefore, such equations are first analyzed from the point of view of asymptotic methods in order to obtain the most efficient initial approximation, and then the well-known methods of numerical integration are applied. The most famous methods of asymptotic analysis equations with rapidly oscillating coefficients are the
Feschenko-Shkil-Nikolenko splitting method $[1,2,3]$ and the Lomov regularization method $[4,5,6,7]$. The first method was applied only to differential systems without an integral operator, while the second allows one to study differential problems in the presence of an integral term (see, for example, $[8,9,10,11,12,13,14,15,16,17,18])$. In this article, Lomov's regularization method is generalized to previously unexplored integrodifferential equations with rapidly oscillating coefficients and rapidly decreasing kernels of the form

$$
\varepsilon \frac{d z}{d t}-A(t) z-\varepsilon g(t) \cos \frac{\beta(t)}{\varepsilon} B(t) z-
$$




$$
\begin{gathered}
-\int_{t_{0}}^{t} e^{\frac{1}{\varepsilon} \int_{s}^{t} \mu(\theta) d \theta} K(t, s) z(s, \varepsilon) d s=h(t), \\
z\left(t_{0}, \varepsilon\right)=z^{0}, t \in\left[t_{0}, T\right],
\end{gathered}
$$

where $\quad z=\left\{z_{1}, z_{2}\right\}, h(t)=\left\{h_{1}(t), h_{2}(t)\right\}, \omega(t)>0$, $\mu(t)>0\left(\forall t \in\left[t_{0}, T\right]\right), g(t)$ is the scalar function, $A(t)$ and $B(t)$ are $(2 \times 2)$-matrices, moreover $A(t)=\left(\begin{array}{cc}0 & 1 \\ -\omega^{2}(t) & 0\end{array}\right), \quad \beta^{\prime}(t)>0$ is the frequency of the rapidly oscillating cosine, $z^{0}=\left\{z_{1}^{0}, z_{2}^{0}\right\}, \varepsilon>0$ is a small parameter. It is precisely such a system in the case $\beta(t)=2 \gamma(t), B(t)=\left(\begin{array}{ll}0 & 0 \\ 1 & 0\end{array}\right)$ and of the absence of an integral term that was considered in $[4,5,6,7]$. The functions $\lambda_{1}(t)=-i \omega(t), \quad \lambda_{2}(t)=+i \omega(t)$ form the spectrum of the limit operator $A(t)$, the function $\lambda_{3}(t) \equiv \mu(t)$ characterizes the rapid change in the kernel of the integral operator, and the functions $\lambda_{4}(t)=-i \beta^{\prime}(t)$ and $\lambda_{5}(t)=+i \beta^{\prime}(t)$ are associated with the presence of a rapidly oscillating cosine in system (1). Previously, such systems have not been considered. This paper is devoted to the generalization of the Lomov's regularization method [4] to systems of the type (1).

Problem (1) will be considered under the following conditions:

$$
\begin{aligned}
& \text { 1) } \omega(t), \mu(t), \beta(t) \in C^{\infty}\left(\left[t_{0}, T\right], \mathrm{R}\right), \\
& g(t) \in\left(\left[t_{0}, T\right], \mathrm{C}^{1}\right), h(t) \in C^{\infty}\left(\left[t_{0}, T\right], \mathrm{C}^{2}\right), \\
& B(t) \in C^{\infty}\left(\left[t_{0}, T\right], \mathrm{C}^{2 \times 2}\right), \\
& K(t, s) \in C^{\infty}\left(\left\{t_{0} \leq s \leq t \leq T\right\}, \mathrm{C}^{2 \times 2}\right) ; \\
& \text { 2) } \omega(t)=\beta^{\prime}(t) \forall t \in\left[t_{0}, T\right](\text { i.e. } \\
& \left.\lambda_{4}(t) \equiv \lambda_{1}(t), \lambda_{5}(t) \equiv \lambda_{2}(t)\right) ;
\end{aligned}
$$

3) the relations

$$
(m, \lambda(t))=0,(m, \lambda(t))=\lambda_{j}(t), j \in\{1,2,3\} \quad \text { for }
$$

all multi-indices $m=\left(m_{1}, m_{2}, m_{3}\right) \quad$ with $|m| \equiv m_{1}+m_{2}+m_{3} \geq 2\left(m_{j} \geq 0, j=1,2,3\right)$ or are not fulfilled for any $t \in\left[t_{0}, T\right]$, or are fulfilled identically on the whole segment $\left[t_{0}, T\right]$.

Here we denoted:

$$
\begin{aligned}
& \lambda(t) \equiv\left(\lambda_{1}(t), \lambda_{2}(t), \lambda_{3}(t)\right), \\
& (m, \lambda(t)) \equiv \sum_{j=1}^{3} m_{j} \lambda_{j}(t)
\end{aligned}
$$

It is clear that under condition 3) resonant multi-indices are exhausted by the following sets:

$$
\begin{gathered}
\Gamma_{0}=\left\{m:(m, \lambda(t)) \equiv 0,|m| \geq 2, \forall t \in\left[t_{0}, T\right]\right\}, \\
\Gamma_{j}=\left\{m:(m, \lambda(t)) \equiv \lambda_{j}(t),|m| \geq 2, \forall t \in\left[t_{0}, T\right]\right\}, \\
j=\overline{1,3} .
\end{gathered}
$$

\subsection{Preliminary considerations}

Functions

$$
v_{3} \equiv e^{-\frac{i}{\varepsilon} \int_{t_{0}}^{t} \beta^{\prime}(\theta) d \theta}, v_{4} \equiv e^{+\frac{i}{\varepsilon} \int_{t_{0}}^{t} \beta^{\prime}(\theta) d \theta},
$$
participating in a rapidly oscillating cosine satisfy differential equations

$$
\begin{gathered}
\varepsilon \frac{d v_{3}}{d t}=-i \beta^{\prime}(t) v_{3}, \varepsilon \frac{d v_{4}}{d t}=+i \beta^{\prime}(t) v_{4}, \\
v_{j}\left(t_{0}\right)=1, j=3,4 .
\end{gathered}
$$

By adding them to system (1), we obtain the problem

$$
\begin{gathered}
\varepsilon \frac{d v}{d t}-A_{1}(t) v-\varepsilon \frac{g(t)}{2}\left(v_{3} \sigma_{1}+v_{4} \sigma_{2}\right) B_{1}(t) v- \\
-\int_{t_{0}}^{t} e^{\frac{1}{\varepsilon} \int_{s}^{t} \mu(\theta) d \theta} K_{1}(t, s) v(s, \varepsilon) d s=H(t) \\
v\left(t_{0}, \varepsilon\right)=v^{0}, t \in\left[t_{0}, T\right]
\end{gathered}
$$

where the notations are introduced:

$$
\begin{array}{r}
v=\left\{z_{1}, z_{2}, v_{3}, v_{4}\right\}, \quad H(t)=\left\{h_{1}(t), h_{2}(t), 0,0\right\}, \\
A_{1}(t)=\left(\begin{array}{cc}
A(t) & 0_{2} \\
0_{2} & \Lambda_{1}(t)
\end{array}\right), B_{1}(t)=\left(\begin{array}{cc}
B(t) & 0_{2} \\
0_{2} & 0_{2}
\end{array}\right), \\
\Lambda_{1}(t)=\left(\begin{array}{cc}
-i \beta^{\prime}(t) & 0 \\
0 & +i \beta^{\prime}(t)
\end{array}\right), \quad 0_{2}=\left(\begin{array}{ll}
0 & 0 \\
0 & 0
\end{array}\right), \\
K_{1}(t, s)=\left(\begin{array}{cc}
K(t, s) & 0_{2} \\
0_{2} & 0_{2}
\end{array}\right), \sigma_{1}=e^{\frac{-i \beta\left(t_{0}\right)}{\varepsilon}}, \sigma_{2}=e^{\frac{+i \beta\left(t_{0}\right)}{\varepsilon}} .
\end{array}
$$

Thus, if $z=\left\{z_{1}(t, \varepsilon), z_{2}(t, \varepsilon)\right\}$ is a solution of the system (1), then $v=\left\{z_{1}(t, \varepsilon), z_{2}(t, \varepsilon), v_{3}(t, \varepsilon)\right.$, $\left.v_{4}(t, \varepsilon)\right\}$ is a solution to a weakly nonlinear system $\left(1_{*}\right)$, and vice versa. Hence, all singularities in the solutions of both systems are described by the spectrum of the matrix $A_{1}(t)$. This matrix will be called the matrix of the extended limit operator of the problem (1). It would be possible to carry out an asymptotic 
analysis of the problem $\left(1_{*}\right)$ instead of the analysis of problem (1), but when developing the corresponding algorithm of the regularization method, this will lead to complex calculations. Therefore, we do not go over to the system $\left(1_{*}\right)$, but consider directly the system (1).

Previously, we considered the case of a simple spectrum of the matrix $A_{1}(t)$, i.e., the case $\omega(t) \neq \beta^{\prime}(t) \forall t \in\left[t_{0}, T\right]$. Here we consider a more complicated case $\omega(t)=\beta^{\prime}(t) \forall t \in\left[t_{0}, T\right]$. In this case, the spectrum of the matrix $A_{1}(t)$ will be multiple and, according to [19] (section 3.6), the algorithm of the regularization method will change significantly.

\section{Regularization of the problem (1)}

We denote, as above, by $\sigma_{j}=\sigma_{j}(\varepsilon)$ independent on $t$ the quantities $\sigma_{1}=e^{-\frac{i}{\varepsilon} \beta\left(t_{0}\right)}$, $\sigma_{2}=e^{+\frac{i}{\varepsilon} \beta\left(t_{0}\right)}$, and rewrite system (1) in the form

$$
\begin{gathered}
\varepsilon \frac{d z}{d t}-A(t) z-\int_{t_{0}}^{t} e^{\frac{1}{\varepsilon} \int_{s}^{t} \mu(\theta) d \theta} K(t, s) z(s, \varepsilon) d s- \\
-\varepsilon \frac{g(t)}{2}\left(e^{-\frac{i}{\varepsilon} \int_{t_{0}}^{t} \beta^{\prime}(\theta) d \theta} \sigma_{1}+e^{+\frac{i}{\varepsilon} \int_{t_{0}}^{t} \beta^{\prime}(\theta) d \theta} \sigma_{2}\right) B(t) z= \\
=h(t), z\left(t_{0}, \varepsilon\right)=z^{0}, t \in\left[t_{0}, T\right] .
\end{gathered}
$$

We introduce regularizing variables (see [4])

$$
\tau_{j}=\frac{1}{\varepsilon} \int_{t_{0}}^{t} \lambda_{j}(\theta) d \theta \equiv \frac{\psi_{j}(t)}{\varepsilon}, j=\overline{1,3}
$$

and instead of problem (2) we consider the problem

$$
\begin{gathered}
\varepsilon \frac{\partial \tilde{z}}{\partial t}+\sum_{j=1}^{3} \lambda_{j}(t) \frac{\partial \tilde{z}}{\partial \tau_{j}}-A(t) \tilde{z}- \\
-\varepsilon \frac{g(t)}{2}\left(e^{\tau_{1}} \sigma_{1}+e^{\tau_{2}} \sigma_{2}\right) B(t) \tilde{z}- \\
-\int_{t_{0}}^{t} e^{\frac{1}{\varepsilon} \int_{s}^{t} \lambda_{3}(\theta) d \theta} K(t, s) \tilde{z}\left(s, \frac{\psi(s)}{\varepsilon}, \varepsilon\right) d s= \\
=h(t),\left.\tilde{z}(t, \tau, \varepsilon)\right|_{t=t_{0}, \tau=0}=z^{0}, t \in\left[t_{0}, T\right],
\end{gathered}
$$

for the function $\tilde{z}=\tilde{z}(t, \tau, \varepsilon)$, where it is indicated (according to (3)): $\tau=\left(\tau_{1}, \tau_{2}, \tau_{3}\right), \psi=\left(\psi_{1}, \psi_{2}, \psi_{3}\right)$. It is clear that if $\tilde{z}=\tilde{z}(t, \tau, \varepsilon)$ is the solution to problem (4), then the vector function $z=\tilde{z}\left(t, \frac{\psi(t)}{\varepsilon}, \varepsilon\right)$ is an exact solution to problem (2), therefore, the problem (4) is expansion of the problem (2). However, it cannot be considered completely regularized, since the integral term

$$
\begin{aligned}
J \tilde{y} & \equiv J\left(\left.\tilde{z}(t, \tau, \varepsilon)\right|_{t=s, \tau=\psi(s) / \varepsilon}\right)= \\
& =\int_{t_{0}}^{t} K(t, s) \tilde{z}\left(s, \frac{\psi(s)}{\varepsilon}, \varepsilon\right) d s
\end{aligned}
$$

has not been regularized in it. To regularize it, we introduce a class $M_{\varepsilon}$, asymptotically invariant with respect to the operator $J \tilde{z}$ (see [4]; p. 62]). We first consider the space of vector functions $z(t, \tau)$, representable by sums

$$
\begin{aligned}
& z(t, \tau, \sigma)=z_{0}(t, \sigma)+\sum_{i=1}^{3} z_{i}(t, \sigma) e^{\tau_{i}}+ \\
& +\sum_{2 \leq|m| \leq N_{z}}^{*} z^{m}(t, \sigma) e^{(m, \tau)}, z_{i}(t, \sigma), z^{m}(t, \sigma) \in \\
& \in C^{\infty}\left(\left[t_{0}, T\right], \mathrm{C}^{2}\right), i=\overline{0,3}, 2 \leq|m| \leq N_{z},
\end{aligned}
$$

where the asterisk * above the sum sign indicates that in it the summation for $|m| \geq 2$ occurs only over nonresonant multi-indices $m=\left(m_{1}, m_{2}, m_{3}\right)$, i.e. over $m \notin \bigcup_{i=0}^{3} \Gamma_{i}$. Note that in (5) the degree of the polynomial with respect to exponentials $e^{\tau_{j}}$ depends on the element $z$. In addition, the elements of space $U$ depend on constant constants $\sigma_{1}=\sigma_{1}(\varepsilon)$ and $\sigma_{2}=\sigma_{2}(\varepsilon)$, which do not affect the development of the algorithm described below, therefore, henceforth, in the notation of element (5) of this space $U$, we omit the dependence on $\sigma=\left(\sigma_{1}, \sigma_{2}\right)$ for brevity. We show that the class $M_{\varepsilon}=\left.U\right|_{\tau=\psi(t) / \varepsilon}$ is asymptotically invariant with respect to the operator $J$.

The image of the operator $J$ on the element (5) of the space $U$ has the form $\left(e_{3}=(0,0,1)\right)$ :

$$
\begin{gathered}
J z(t, \tau)=\int_{t_{0}}^{t} e^{\frac{1}{\varepsilon} \int_{s}^{t} \lambda_{3}(\theta) d \theta} K(t, s) z_{0}(s) d s+ \\
+\sum_{i=1}^{3} \int_{t_{0}}^{t} e^{\frac{1}{\varepsilon} \int_{s}^{t} \lambda_{3}(\theta) d \theta} K(t, s) z_{i}(s) e^{\frac{1}{\varepsilon} \int_{t_{0}}^{s} \lambda_{i}(\theta) d \theta} d s+
\end{gathered}
$$




$$
\begin{gathered}
+\sum_{2 \leq|m| \leq N_{z}}^{*} \int_{t_{0}}^{t} e^{\frac{1}{\varepsilon} \int_{s}^{t} \lambda_{3}(\theta) d \theta} K(t, s) z^{m}(s) e^{\frac{1}{\varepsilon} \int_{t_{0}}^{s}(m, \lambda(\theta)) d \theta} d s= \\
=\int_{t_{0}}^{t} e^{\frac{1}{\varepsilon} \int_{s}^{t} \lambda_{3}(\theta) d \theta} K(t, s) z_{0}(s) d s+ \\
+\sum_{i=1}^{2} e^{\frac{1}{\varepsilon} \int_{t_{0}}^{t} \lambda_{3}(\theta) d \theta} \int_{t_{0}}^{t} K(t, s) z_{i}(s) e^{\frac{1}{\varepsilon} \int_{t_{0}}^{s}\left(\lambda_{i}(\theta)-\lambda_{3}(\theta)\right) d \theta} d s+ \\
+\sum_{2 \leq|m| \leq N_{z}}^{+\frac{1}{\varepsilon} \int_{t_{0}}^{t} K(t, s) z_{3}(s) d s+} d s .
\end{gathered}
$$

Integrating by parts, we have

$$
\begin{gathered}
J_{0}(t, \varepsilon)=\int_{t_{0}}^{t} K(t, s) z_{0}(s) e^{\frac{1}{\varepsilon} \int_{s}^{t} \lambda_{3}(\theta) d \theta} d s= \\
=\varepsilon \int_{t_{0}}^{t} \frac{K(t, s) z_{0}(s)}{-\lambda_{3}(s)} d e^{\frac{1}{\varepsilon} \int_{s}^{t} \lambda_{3}(\theta) d \theta}= \\
=\left.\varepsilon \frac{K(t, s) z_{0}(s)}{-\lambda_{3}(s)} e^{\frac{1}{\varepsilon} \int_{s}^{t} \lambda_{3}(\theta) d \theta}\right|_{s=t} ^{s=t}- \\
-\varepsilon \int_{t_{0}}^{t}\left(\frac{\partial}{\partial s} \frac{K(t, s) z_{0}(s)}{-\lambda_{3}(s)}\right) e^{\frac{1}{\varepsilon} \int_{s}^{t} \lambda_{3}(\theta) d \theta} d s= \\
=\varepsilon\left[\frac{K\left(t, t_{0}\right) z_{0}\left(t_{0}\right)}{\lambda_{3}\left(t_{0}\right)} e^{\frac{1}{\varepsilon} \int_{t_{0}}^{t} \lambda_{3}(\theta) d \theta}-\frac{K(t, t) z_{0}(t)}{\lambda_{3}(t)}\right]+ \\
+\varepsilon \int_{t_{0}}^{t}\left(\frac{\partial}{\partial s} \frac{K(t, s) z_{0}(s)}{\lambda_{3}(s)}\right) e^{\frac{1}{\varepsilon} \int_{t_{0}}^{t} \lambda_{3}(\theta) d \theta} d s .
\end{gathered}
$$

Continuing this process further, we obtain the decomposition

$$
\begin{gathered}
J_{0}(t, \varepsilon)=\sum_{v=0}^{\infty} \varepsilon^{v+1}\left[\left(I_{0}^{v}\left(K(t, s) z_{0}(s)\right)\right)_{s=t_{0}} e^{\frac{1}{\varepsilon} \int_{t_{0}}^{t} \lambda_{3}(\theta) d \theta}-\right. \\
\left.-\left(I_{0}^{v}\left(K(t, s) z_{0}(s)\right)\right)_{s=t}\right], \\
I_{0}^{0}=\frac{1}{\lambda_{3}(s)}, I_{0}^{v}=\frac{1}{\lambda_{3}(s)} \frac{\partial}{\partial s} I_{0}^{v-1}(v \geq 1) .
\end{gathered}
$$

Next, apply the same operation to the integrals:

$$
\begin{gathered}
J_{3, i}(t, \varepsilon)=e^{\frac{1}{\varepsilon} \int_{t_{0}}^{t} \lambda_{3}(\theta) d \theta} \int_{t_{0}}^{t} K(t, s) z_{i}(s) \times \\
\times e^{\frac{1}{\varepsilon} \int_{t_{0}}^{s}\left(\lambda_{i}(\theta)-\lambda_{3}(\theta)\right) d \theta} d s= \\
=\varepsilon e^{\frac{1}{\varepsilon} \int_{t_{0}}^{t} \lambda_{3}(\theta) d \theta} \int_{t_{0}}^{t} \frac{K(t, s) z_{i}(s)}{\lambda_{i}(s)-\lambda_{3}(s)} d e^{\frac{1}{\varepsilon} \int_{t_{0}}^{s}\left(\lambda_{i}(\theta)-\lambda_{3}(\theta)\right) d \theta}=
\end{gathered}
$$

$$
\begin{gathered}
=\varepsilon e^{\frac{1}{\varepsilon} \int_{t_{0}}^{t} \lambda_{3}(\theta) d \theta}\left[\left.\frac{K(t, s) z_{i}(s)}{\lambda_{i}(s)-\lambda_{3}(s)} e^{\frac{1}{\varepsilon} \int_{t_{0}}^{t}\left(\lambda_{i}(\theta)-\lambda_{3}(\theta)\right) d \theta}\right|_{s=t_{0}} ^{s=t}-\right. \\
-\varepsilon \int_{t_{0}}^{t}\left(\frac{\partial}{\partial s} \frac{K(t, s) z_{i}(s)}{\lambda_{j}(s)-\lambda_{3}(s)}\right) e^{\frac{1}{\varepsilon} \int_{t_{0}}^{t}\left(\lambda_{i}(\theta)-\lambda_{3}(\theta)\right) d \theta} d s \mid= \\
=\varepsilon\left\lfloor\frac{K(t, t) z_{i}(t)}{\lambda_{i}(t)-\lambda_{3}(t)} e^{\frac{1}{\varepsilon} \int_{t_{0}}^{t} \lambda_{i}(\theta) d \theta}-\right. \\
\left.-\frac{K\left(t, t_{0}\right) z_{i}\left(t_{0}\right)}{\lambda_{i}\left(t_{0}\right)-\lambda_{3}\left(t_{0}\right)} e^{\frac{1}{\varepsilon} \int_{t_{0}}^{t} \lambda_{3}(\theta) d \theta}\right\rfloor- \\
-\varepsilon e^{\frac{1}{\varepsilon} \int_{t_{0}}^{t} \lambda_{3}(\theta) d \theta} \int_{t_{0}}^{t}\left(\frac{\partial}{\partial s} \frac{K(t, s) z_{i}(s)}{\lambda_{i}(s)-\lambda_{3}(s)}\right) \times \\
\frac{1}{\varepsilon} \int_{t_{0}}^{s}\left(\lambda_{i}(\theta)-\lambda_{3}(\theta)\right) d \theta \\
\times e^{d s}=
\end{gathered}
$$$$
=\sum_{v=0}^{\infty}(-1)^{v} \varepsilon^{v+1}\left[\left(I_{i}^{v}\left(K(t, s) z_{i}(s)\right)\right)_{s=t} e^{\left.\frac{1}{\varepsilon} \int_{t_{0}}^{t} \lambda_{i}(\theta)\right) d \theta}-\right.
$$$$
\left.-\left(I_{i}^{v}\left(K(t, s) z_{i}(s)\right)\right)_{s=t_{0}} e^{\frac{1}{\varepsilon} \int_{t_{0}}^{t} \lambda_{3}(\theta) d \theta}\right],
$$$$
I_{i}^{0}=\frac{1}{\lambda_{i}(s)-\lambda_{3}(s)}, I_{i}^{v}=\frac{1}{\lambda_{i}(s)-\lambda_{3}(s)} \frac{\partial}{\partial s} I_{i}^{v-1},
$$$$
i=1,2 \text {; }
$$$$
J_{m}(t, \varepsilon)=e^{\frac{1}{\varepsilon} \int_{t_{0}}^{t} \lambda_{3}(\theta) d \theta} \int_{t_{0}}^{t} K(t, s) z^{m}(s) \times
$$$$
\times e^{\frac{1}{\varepsilon} \int_{t_{0}}^{s}\left(m-e_{3}, \lambda(\theta)\right) d \theta} d s=
$$$$
=\varepsilon e^{\frac{1}{\varepsilon} \int_{t_{0}}^{t} \lambda_{3}(\theta) d \theta} \int_{t_{0}}^{t} \frac{K(t, s) z^{m}(s)}{\left(m-e_{3}, \lambda(s)\right)} d e^{\frac{1}{\varepsilon} \int_{t_{0}}^{s}\left(m-e_{3}, \lambda(\theta)\right) d \theta}=
$$$$
=\varepsilon e^{\frac{1}{\varepsilon} \int_{t_{0}}^{t} \lambda_{3}(\theta) d \theta}\left[\left.\frac{K(t, s) z^{m}(s)}{\left(m-e_{3}, \lambda(s)\right)} e^{\frac{1}{\varepsilon} \int_{t_{0}}^{t}\left(m-e_{3}, \lambda(\theta)\right) d \theta}\right|_{s=t_{0}} ^{s=t}-\right.
$$$$
\left.-\int_{t_{0}}^{t}\left(\frac{\partial}{\partial s} \frac{K(t, s) z^{m}(s)}{\left(m-e_{3}, \lambda(s)\right)}\right) e^{\frac{1}{\varepsilon} \int_{t_{0}}^{s}\left(m-e_{3}, \lambda(\theta)\right) d \theta} d s\right]=
$$$$
=\varepsilon\left\lfloor\frac{K(t, t) z^{m}(t)}{\left(m-e_{3}, \lambda(t)\right)} e^{\frac{1}{\varepsilon} \int_{t_{0}}^{t}(m, \lambda(\theta)) d \theta}-\right.
$$$$
\left.-\frac{K\left(t, t_{0}\right) z^{m}\left(t_{0}\right)}{\left(m-e_{3}, \lambda\left(t_{0}\right)\right)} e^{\frac{1}{\varepsilon} \int_{t_{0}}^{t} \lambda_{3}(\theta) d \theta}\right]-
$$ 


$$
\begin{gathered}
-\varepsilon e^{\frac{1}{\varepsilon} \int_{t_{0}}^{t} \lambda_{3}(\theta) d \theta} \int_{t_{0}}^{t}\left(\frac{\partial}{\partial s} \frac{K(t, s) z^{m}(s)}{\left(m-e_{3}, \lambda(s)\right)}\right) \times \\
=\sum_{v=0}^{\infty}(-1)^{v} \varepsilon^{v+1}\left[\left(I_{3, m}^{v}\left(K(t, s) z^{m}(s)\right)\right)_{s=t} \times\right. \\
\left.-\left(I_{3, m}^{v}\left(K(t, s) z^{m}(s)\right)\right)_{s=t_{0}} e^{\left.\frac{1}{\varepsilon} \int_{t_{0}}^{t} \lambda_{3}(\theta) d \theta\right) d \theta}\right], \\
\times e^{\frac{1}{\varepsilon} \int_{t_{0}}^{t}(m, \lambda(\theta)) d \theta}, \\
I_{3, m}^{v}=\frac{1}{\left(m-e_{3}, \lambda(s)\right)} \frac{\partial}{\partial s} I_{3, m}^{v-1}, 2 \leq|m| \leq N_{z} .
\end{gathered}
$$

Here it is taken into account that since $\left(m-e_{3}, \lambda(s)\right) \neq 0$, since by definition of space $U$, multi-indices $m \notin \Gamma_{3}$. This means that the image of the operator $J$ on the element (5) of the space $U$ is represented as a series

$$
\begin{aligned}
& J z(t, \tau)=e^{\left.\frac{1}{\varepsilon} \int_{t_{0}}^{t} \lambda_{3}(\theta)\right) d \theta} \int_{t_{0}}^{t} K(t, s) z_{3}(s) d s+ \\
& +\sum_{\nu=0}^{\infty} \varepsilon^{v+1}\left\lfloor\left(I_{0}^{v}\left(K(t, s) z_{0}(s)\right)\right)_{s=t_{0}} e^{\left.\frac{1}{\varepsilon} \int_{t_{0}}^{t} \lambda_{3}(\theta)\right) d \theta}-\right. \\
& \left.-\left(I_{0}^{v}\left(K(t, s) z_{0}(s)\right)\right)_{s=t}\right\rfloor+ \\
& +\sum_{i=1}^{2} \sum_{v=0}^{\infty}(-1)^{v} \varepsilon^{v+1}\left[\left(I_{i}^{v}\left(K(t, s) z_{i}(s)\right)\right)_{s=t} \times\right. \\
& \times e^{\left.\frac{1}{\varepsilon} \int_{t_{0}}^{t} \lambda_{i}(\theta)\right) d \theta} \\
& \left.\left.-\left(I_{i}^{v}\left(K(t, s) z_{i}(s)\right)\right)\right)_{s=t_{0}} e^{\frac{1}{\varepsilon} \int_{t_{0}}^{t} \lambda_{3}(\theta) d \theta}\right\rfloor+ \\
& +\sum_{2 \leq m \mid \leq N}^{*} \sum_{z=0}^{\infty}(-1)^{v} \varepsilon^{v+1}\left[\left(I_{3, m}^{v}\left(K(t, s) z^{m}(s)\right)\right)_{s=t} \times\right. \\
& \times e^{\frac{1}{\varepsilon} \int_{t_{0}}^{t}(m, \lambda(\theta)) d \theta} \\
& \left.-\left(I_{3, m}^{v}\left(K(t, s) z^{m}(s)\right)\right)_{s=t_{0}} e^{\frac{1}{\varepsilon} \int_{t_{0}}^{t} \lambda_{3}(\theta) d \theta}\right] .
\end{aligned}
$$

It is easy to show (see, for example, [19], pages 291-294) that this series converges asymptotically for $\varepsilon \rightarrow+0$ (uniformly in $\left.t \in\left[t_{0}, T\right]\right)$. This means that the class $M_{\varepsilon}$ is asymptotically invariant (as $\varepsilon \rightarrow+0$ ) with respect to the operator $J$.

We introduce the operators $R_{v}: U \rightarrow U$, acting on each element $z(t, \tau) \in U$ of the form (5) according to the law:

$$
\begin{gathered}
R_{0} z(t, \tau)=e^{\tau_{3}} \int_{t_{0}}^{t} K(t, s) z_{3}(s) d s, \quad\left(6_{0}\right) \\
R_{1} z(t, \tau)=\left[\left(I_{0}^{0}\left(K(t, s) z_{0}(s)\right)\right)_{s=t_{0}} e^{\tau_{3}}-\right. \\
\left.-\left(I_{0}^{0}\left(K(t, s) z_{0}(s)\right)\right)_{s=t}\right]+ \\
+\sum_{i=1}^{2}\left[\left(I_{i}^{0}\left(K(t, s) z_{i}(s)\right)\right)_{s=t} e^{\tau_{i}}-\right. \\
\left.-\left(I_{i}^{0}\left(K(t, s) z_{i}(s)\right)\right)_{s=t_{0}} e^{\tau_{3}}\right]+ \\
+\sum_{2 \leq|m| \leq N_{z}}^{*}\left[\left(I_{3, m}^{0}\left(K(t, s) z^{m}(s)\right)\right)_{s=t} e^{(m, \tau)}-\right. \\
\left.-\left(I_{3, m}^{0}\left(K(t, s) z^{m}(s)\right)\right)_{s=t_{0}} e^{\tau_{3}}\right], \\
R_{v+1} z(t, \tau)=\left[\left(I_{0}^{v}\left(K(t, s) z_{0}(s)\right)\right)_{s=t_{0}} e^{\tau_{3}}-\right. \\
\left.-\left(I_{0}^{v}\left(K(t, s) z_{0}(s)\right)\right)_{s=t}\right]+ \\
+\sum_{i=1}^{2}(-1)^{v}\left[\left(I_{i}^{v}\left(K(t, s) z_{i}(s)\right)\right)_{s=t} e^{\tau_{i}}-\right. \\
\left.\left.-\left(I_{i}^{v}\left(K(t, s) z_{i}(s)\right)\right)\right)_{s=t_{0}} e^{\tau_{3}}\right]+ \\
+\sum_{2 \leq m \mid \leq N_{z}}^{*}\left[\left(I_{3, m}^{v}\left(K(t, s) z^{m}(s)\right)\right)_{s=t} e^{(m, \tau)}-\right. \\
\left.-\left(I_{3, m}^{v}\left(K(t, s) z^{m}(s)\right)\right)_{s=t_{0}} e^{\tau_{3}}\right] .
\end{gathered}
$$

Let now $\tilde{z}(t, \tau, \varepsilon)$ be an arbitrary continuous function in $(t, \tau) \in\left[t_{0}, T\right] \times\left\{\tau: \operatorname{Re} \tau_{j} \leq 0, j=\overline{1,3}\right\}$ with asymptotic expansion

$$
\tilde{z}(t, \tau, \varepsilon)=\sum_{k=0}^{\infty} \varepsilon^{k} z_{k}(t, \tau), z_{k}(t, \tau) \in U,
$$

converging as $\varepsilon \rightarrow+0$ (uniformly in $\left.(t, \tau) \in\left[t_{0}, T\right] \times\left\{\tau: \operatorname{Re\tau }_{j} \leq 0, j=\overline{1,3}\right\}\right)$. Then the image $J \tilde{z}(t, \tau, \varepsilon)$ of this function is decomposed into an asymptotic series

$$
J \tilde{z}(t, \tau, \varepsilon)=\sum_{k=0}^{\infty} \varepsilon^{k} J z_{k}(t, \tau)=
$$




$$
=\left.\sum_{r=0}^{\infty} \varepsilon^{r} \sum_{s=0}^{r} R_{r-s} z_{s}(t, \tau)\right|_{\tau=\psi(t) / \varepsilon} .
$$

This equality is the basis for introducing an extension of the operator $J$ on series of the form (7):

$$
\begin{aligned}
\tilde{J} \tilde{z}(t, \tau, \varepsilon) & \equiv \tilde{J}\left(\sum_{k=0}^{\infty} \varepsilon^{k} z_{k}(t, \tau)\right) \stackrel{\text { def }}{=} \\
& \stackrel{\operatorname{def}}{=} \sum_{r=0}^{\infty} \varepsilon^{r} \sum_{s=0}^{r} R_{r-s} z_{s}(t, \tau) .
\end{aligned}
$$

Although the operator $\tilde{J}$ is formally defined, its usefulness is obvious, since in practice it is usual to construct the $N$-th approximation of the asymptotic solution of problem (2), in which only $N$-th partial sums of the series (7) will take part, which have not formal, but true meaning. Now we can write down a problem that is completely regularized with respect to the original problem (2):

$$
\begin{gathered}
\varepsilon \frac{\partial \tilde{z}}{\partial t}+\sum_{j=1}^{3} \lambda_{j}(t) \frac{\partial \tilde{z}}{\partial \tau_{j}}-A(t) \tilde{z}- \\
-\varepsilon \frac{g(t)}{2}\left(e^{\tau_{1}} \sigma_{1}+e^{\tau_{2}} \sigma_{2}\right) B \tilde{z}-\tilde{J} \tilde{z}= \\
=h(t),\left.\tilde{z}(t, \tau, \varepsilon)\right|_{t=t_{0}, \tau=0}=z^{0}, t \in\left[t_{0}, T\right],
\end{gathered}
$$

where the operator has the form (8).

\section{Iterative problems and their solvability in the space $U$. Solution of the first iterative problem}

Substituting the series (7) into (9) and equating the coefficients for the same powers of $\varepsilon$, we obtain the following iterative problems:

$$
\begin{aligned}
& L z_{0}(t, \tau) \equiv \sum_{j=1}^{3} \lambda_{j}(t) \frac{\partial z_{0}}{\partial \tau_{j}}-A(t) z_{0}-R_{0} z_{0}= \\
&= h(t), z_{0}\left(t_{0}, 0\right)=z^{0} ; \\
& L z_{1}(t, \tau)=-\frac{\partial z_{0}}{\partial t}+\frac{g(t)}{2}\left(e^{\tau_{1}} \sigma_{1}+e^{\tau_{2}} \sigma_{2}\right) \times \\
& \times B(t) z_{0}+R_{1} z_{0}, z_{1}\left(t_{0}, 0\right)=0 ; \\
& L z_{2}(t, \tau)=-\frac{\partial z_{1}}{\partial t}+\frac{g(t)}{2}\left(e^{\tau_{1}} \sigma_{1}+e^{\tau_{2}} \sigma_{2}\right) \times \\
& \times B(t) z_{1}+R_{1} z_{1}+R_{2} z_{0}, z_{0}\left(t_{0}, 0\right)=0 ;
\end{aligned}
$$

$$
\begin{gathered}
L z_{k}(t, \tau)=-\frac{\partial z_{k-1}}{\partial t}+\frac{g(t)}{2}\left(e^{\tau_{1}} \sigma_{1}+e^{\tau_{2}} \sigma_{2}\right) \times \\
\times B(t) z_{k-1}+R_{k} z_{0}+\ldots+R_{1} z_{k-1}, \\
z_{k}\left(t_{0}, 0\right)=0, k \geq 1 .
\end{gathered}
$$

Each of the iterative problem $\left(10_{k}\right)$ can be written as

$$
\begin{aligned}
L z(t, \tau) & \equiv \sum_{j=1}^{3} \lambda_{j}(t) \frac{\partial z}{\partial \tau_{j}}-A(t) z-R_{0} z= \\
& =H(t, \tau), \quad z\left(t_{0}, 0\right)=z^{*},
\end{aligned}
$$

where $\quad H(t, \tau)=H_{0}(t)+\sum_{i=1}^{3} H_{i}(t) e^{\tau_{i}}+$ $+\sum_{2 \leq|m| \leq N_{H}}^{*} H^{m}(t) e^{(m, \tau)}$ is a well-known vectorfunction of the space $U, z^{*}$ is a well-known constant vector of a complex space $\mathrm{C}^{2}$, and the operator $R_{0}$ has the form (see $\left(6_{0}\right)$ )

$$
\begin{aligned}
& R_{0} z \equiv R_{0}\left(z_{0}(t)+\sum_{i=1}^{3} z_{i}(t) e^{\tau_{i}}+\sum_{2 \leq|m| \leq N_{z}}^{*} z^{m}(t) e^{(m, \tau)}\right)= \\
& =e^{\tau_{3}} \int_{t_{0}}^{t} K(t, s) z_{3}(s) d s .
\end{aligned}
$$

In the future we need the $\bar{\lambda}_{j}(t)$-eigenvectors of the matrix $A(t)$ :

$$
\varphi_{1}(t)=\left(\begin{array}{c}
1 \\
-i \omega(t)
\end{array}\right), \varphi_{2}(t)=\left(\begin{array}{c}
1 \\
+i \omega(t)
\end{array}\right),
$$

as well as $\bar{\lambda}_{j}(t)$-eigenvectors of the matrix $A^{*}(t)$ :

$$
\chi_{1}(t)=\frac{1}{2}\left(\begin{array}{c}
1 \\
-\frac{i}{\omega(t)}
\end{array}\right), \chi_{2}(t)=\frac{1}{2}\left(\begin{array}{c}
1 \\
+\frac{i}{\omega(t)}
\end{array}\right) .
$$

These vectors form a biorthogonal system, i.e.

$$
\left(\varphi_{k}(t), \chi_{j}(t)\right)=\left\{\begin{array}{l}
1, k=j, \\
0, k \neq j
\end{array}(k, j=1,2) .\right.
$$

We introduce the scalar product (for each $\left.t \in\left[t_{0}, T\right]\right)$ in the space $U$ :

$$
\begin{gathered}
<z, w>\equiv<z_{0}(t)+\sum_{i=1}^{3} z_{i}(t) e^{\tau_{i}}+\sum_{2 \leq|m| \leq N_{z}}^{*} z^{m}(t) e^{(m, \tau)}, \\
w_{0}(t)+\sum_{i=1}^{3} w_{i}(t) e^{\tau_{i}}+\sum_{2 \leq|m| \leq N_{w}}^{*} w^{m}(t) e^{(m, \tau)}>\stackrel{\text { def }}{=} \\
\stackrel{\text { def }}{=}\left(z_{0}(t), w_{0}(t)\right)+\sum_{i=1}^{3}\left(z_{i}(t), w_{i}(t)\right)+ \\
+\sum_{2 \leq|m| \leq \min \left(N_{z}, N_{w}\right)}^{*}\left(z^{m}(t), w^{m}(t)\right),
\end{gathered}
$$


where we denote by $(*, *)$ the ordinary scalar product in a complex space $\square^{2}$. We prove the following statement.

Theorem 1. Let conditions 1) and 2) are satisfied and the right-hand side $H(t, \tau)=H_{0}(t)+\sum_{i=1}^{3} H_{i}(t) e^{\tau_{i}}+\sum_{2 \leq|m| \leq N_{H}}^{*} H^{m}(t) e^{(m, \tau)}$

of the system (11) belongs to the space $U$. Then for the solvability of system (11) in $U$ it is necessary and sufficient that the identities

$$
<H(t, \tau), \chi_{k}(t) e^{\tau_{k}}>\equiv 0, k=1,2, \forall t \in\left[t_{0}, T\right]
$$
hold.

Proof. We will determine the solution to system (11) in the form of an element (5) of the space $U$ :

$$
z(t, \tau)=z_{0}(t)+\sum_{i=1}^{3} z_{i}(t) e^{\tau_{i}}+\sum_{2 \leq|m| \leq N_{H}}^{*} z^{m}(t) e^{(m, \tau)}
$$

Substituting (13) into the system (11), we have

$$
\begin{gathered}
\sum_{i=1}^{3}\left[\lambda_{i}(t) I-A(t)\right] z_{i}(t) e^{\tau_{i}}+ \\
+\sum_{2 \leq|m| \leq N_{H}}^{*}[(m, \lambda(t)) I-A(t)] z^{m}(t) e^{(m, \tau)}- \\
-A(t) z_{0}(t)-e^{\tau_{3}} \int_{t_{0}}^{t} K(t, s) z_{3}(s) d s= \\
=H_{0}(t)+\sum_{i=1}^{3} H_{i}(t) e^{\tau_{i}}+\sum_{2 \leq|m| \leq N_{H}}^{*} H^{m}(t) e^{(m, \tau)} .
\end{gathered}
$$

Equating here separately the free terms and coefficients at the same exponents, we obtain the following systems of equations:

$$
\begin{gathered}
-A(t) z_{0}(t)=H_{0}(t), \\
{\left[\lambda_{i}(t) I-A(t)\right] z_{i}(t)=H_{i}(t), i=\overline{1,2} ;} \\
\left\lfloor\lambda_{3}(t) I-A(t)\right\rfloor z_{3}(t)- \\
-\int_{t_{0}}^{t} K(t, s) z_{3}(s) d s=H_{3}(t), \\
{[(m, \lambda(t)) I-A(t)] z^{m}(t)=H^{m}(t),} \\
2 \leq|m| \leq N_{H}, m \notin \bigcup_{j=0}^{3} \Gamma_{j} .
\end{gathered}
$$

Due to the invertibility of the matrix $A(t)$, system $\left(14_{0}\right)$ has a solution $z_{0}(t)=-A^{-1}(t) H_{0}(t)$. Since $\lambda_{3}(t)=\mu(t)$ is a real function, and the eigenvalues of the matrix $A(t)$ are purely imaginary, the matrix $\lambda_{3}(t) I-A(t)$ is invertible and therefore system $\left(14_{3}\right)$ can be written as

$$
\begin{aligned}
& z_{3}(t)=\int_{t_{0}}^{t}\left(\left[\lambda_{3}(t) I-A(t)\right]^{-1} K(t, s)\right) \times \\
& \times z_{3}(s) d s+\left[\lambda_{3}(t) I-A(t)\right]^{-1} H_{3}(t) .
\end{aligned}
$$

Due to the smoothness of the kernel $\left(\left[\lambda_{3}(t) I-A(t)\right]^{-1} K(t, s)\right)$ and the heterogeneity $\quad\left[\lambda_{3}(t) I-A(t)\right]^{-1} H_{3}(t)$ this Volterra integral system has a unique solution $z_{3}(t) \in C^{\infty}\left(\left[t_{0}, T\right], \mathrm{C}^{2}\right)$.

Systems $\left(14_{1}\right)$ and $\left(14_{2}\right)$ are solvable in the space $C^{\infty}\left(\left[t_{0}, T\right], \square^{2}\right)$ if and only if the identities $\left(H_{i}(t), \chi_{i}(t)\right) \equiv 0 \forall t \in\left[t_{0}, T\right], i=1,2$ hold. It is easy to see that these identities coincide with the identities (12). Further, since multi-indices $m \notin \bigcup_{j=0}^{3} \Gamma_{j}$ in the systems $\left(14_{m}\right)$, then $(m, \lambda(t)) \neq \lambda_{i}(t), i=1,2$, so these systems are uniquely solvable in the space $C^{\infty}\left(\left[t_{0}, T\right], \mathrm{C}^{2}\right)$ in the form of functions

$$
\begin{aligned}
z^{m}(t)= & {[(m, \lambda(t)) I-A(t)]^{-1} H^{m}(t), } \\
& 0 \leq|m| \leq N_{H} .
\end{aligned}
$$

Thus, condition (12) is necessary and sufficient for the solvability of system (11) in space $U$. The theorem is proved.

Remark 1. If identity (12) holds, then under conditions 1) and 2) system (11) has (see (15) (16)) the following solution in space $U$ :

$$
\begin{array}{r}
z(t, \tau)=z_{0}(t)+\sum_{i=1}^{3} z_{i}(t) e^{\tau_{i}}+\sum_{2 \leq|m| \leq N_{H}}^{*} z^{m}(t) e^{(m, \tau)} \equiv \\
\equiv z_{0}(t)+\sum_{k=1}^{2} \alpha_{k}(t) \varphi_{k}(t) e^{\tau_{k}}+h_{12}(t) \varphi_{2}(t) e^{\tau_{1}}+(17) \\
+h_{21}(t) \varphi_{1}(t) e^{\tau_{2}}+z_{3}(t) e^{\tau_{3}}+\sum_{2 \leq|m| \leq N_{H}}^{*} P^{m}(t) e^{(m, \tau)},
\end{array}
$$

where $\alpha_{k}(t) \in C^{\infty}\left(\left[t_{0}, T\right], C^{1}\right)$ are arbitrary functions, $\quad k=1,2, z_{0}(t)=-A^{-1} H_{0}(t), z_{3}(t)$ is the solution of the integral system (15) and the notation is introduced: 


$$
\begin{gathered}
h_{12}(t) \equiv \frac{\left(H_{1}(t), \chi_{2}(t)\right)}{\lambda_{1}(t)-\lambda_{2}(t)}, h_{21}(t) \equiv \frac{\left(H_{2}(t), \chi_{1}(t)\right)}{\lambda_{2}(t)-\lambda_{1}(t)}, \\
P_{i}(t) \equiv\left[\lambda_{i}(t) I-A(t)\right]^{-1} H_{i}(t), \\
P^{m}(t) \equiv[(m, \lambda(t)) I-A(t)]^{-1} H^{m}(t) .
\end{gathered}
$$

\section{The unique solvability of the general iterative problem in the space $U$. The remainder term theorem}

We proceed to the description of the conditions for the unique solvability of system (11) in the space $U$. Along with problem (11), we consider the system

$$
\begin{aligned}
L w(t, \tau)= & -\frac{\partial z}{\partial t}+\frac{g(t)}{2}\left(e^{\tau_{1}} \sigma_{1}+e^{\tau_{2}} \sigma_{2}\right) \times \\
& \times B(t) z+R_{1} z+Q(t, \tau),
\end{aligned}
$$

where $z=z(t, \tau)$ is the solution (18) of the system (11), $Q(t, \tau) \in U$ is the known function of the space $U$. The right-hand side of this system:

$$
\begin{gathered}
G(t, \tau) \equiv-\frac{\partial z}{\partial t}+\frac{g(t)}{2}\left(e^{\tau_{1}} \sigma_{1}+e^{\tau_{2}} \sigma_{2}\right) \times \\
\times B(t) z+R_{1} z+Q(t, \tau)= \\
=-\frac{\partial}{\partial t}\left[z_{0}(t)+\sum_{i=1}^{3} z_{i}(t) e^{\tau_{i}}+\sum_{2 \leq|m| \leq N_{z}}^{*} z^{m}(t) e^{(m, \tau)}\right]+ \\
+\frac{g(t)}{2}\left(e^{\tau_{1}} \sigma_{1}+e^{\tau_{2}} \sigma_{2}\right) B(t)\left[z_{0}(t)+\sum_{i=1}^{2} z_{i}(t) e^{\tau_{i}}+\right. \\
\left.+\sum_{2 \leq|m| \leq N_{z}}^{*} z^{m}(t) e^{(m, \tau)}\right\rfloor+R_{1} z+Q(t, \tau)
\end{gathered}
$$

may not belong to the space $U$, if $z=z(t, \tau) \in U$. Since $-\frac{\partial z}{\partial t}, R_{1} z, Q(t, \tau) \in U$, then this fact needs to be checked for the function

$$
\begin{gathered}
Z(t, \tau) \equiv \frac{g(t)}{2}\left(e^{\tau_{1}} \sigma_{1}+e^{\tau_{2}} \sigma_{2}\right) B(t)\left[z_{0}(t)+\right. \\
\left.+\sum_{i=1}^{3} z_{i}(t) e^{\tau_{i}}+\sum_{2 \leq|m| \leq N_{z}}^{*} z^{m}(t) e^{(m, \tau)}\right]=
\end{gathered}
$$

$$
\begin{gathered}
=\frac{g(t)}{2} B(t) z_{0}(t)\left(e^{\tau_{1}} \sigma_{1}+e^{\tau_{2}} \sigma_{2}\right)+ \\
+\sum_{i=1}^{3} \frac{g(t)}{2} B(t) z_{i}(t)\left(e^{\tau_{i}+\tau_{1}} \sigma_{1}+e^{\tau_{i}+\tau_{2}} \sigma_{2}\right)+ \\
+\frac{g(t)}{2}\left(e^{\tau_{1}} \sigma_{1}+e^{\tau_{2}} \sigma_{2}\right) B(t) \sum_{2 \leq|m| \leq N_{H}}^{*} z^{m}(t) e^{(m, \tau)} .
\end{gathered}
$$

Function $Z(t, \tau) \notin U$, since it has resonant exponents

$$
\begin{aligned}
& e^{\tau_{1}+\tau_{2}}=\left.e^{(m, \tau)}\right|_{m=(1,1,0)}, e^{\tau_{1}+(m, \tau)}\left(m_{1}+1=m_{2}, m_{3}=0\right), \\
& e^{\tau_{2}+(m, \tau)}\left(m_{2}+1=m_{1}, m_{3}=0\right),
\end{aligned}
$$

therefore, the right-hand side $G(t, \tau)=Z(t, \tau)-\frac{\partial z}{\partial t}+R_{1} z+Q(t, \tau)$ of $\quad$ system (19) also does not belong to space $U$. Then, according to the well-known theory (see [5], p. 234), it is necessary to embed $\wedge: G(t, \tau) \rightarrow \hat{G}(t, \tau)$ the right-hand side $G(t, \tau)$ of the system (19) in the space $U$. This operation is defined as follows. Let the function $G(t, \tau)=\sum_{|m|=0}^{N} w^{m}(t) e^{(m, \tau)} \quad$ contain resonant exponentials, i.e. $G(t, \tau)$ has the form

$$
\begin{gathered}
G(t, \tau)=w_{0}(t)+\sum_{i=1}^{3} w_{i}(t) e^{\tau_{i}}+ \\
+\sum_{j=0}^{3} \sum_{\left|m^{j}\right|=2: m^{j} \in \Gamma_{j}}^{N} w^{m^{j}}(t) e^{\left(m^{j}, \tau\right)}+ \\
+\sum_{|m|=2, m \neq m^{j}, j=\overline{0,3}}^{N} w^{m}(t) e^{(m, \tau)} .
\end{gathered}
$$

Then

$$
\begin{gathered}
\hat{G}(t, \tau)=w_{0}(t)+\sum_{i=1}^{3} w_{i}(t) e^{\tau_{i}}+ \\
+\sum_{j=0}^{3} \sum_{\left|m^{j}\right|=2: m^{j} \in \Gamma_{j}}^{N} w^{m^{j}}(t) e^{\tau_{j}}+ \\
+\sum_{|m|=2, m \neq m^{j}, j=0,3}^{N} w^{m}(t) e^{(m, \tau)}
\end{gathered}
$$

Therefore, the embedding operation acts only on the resonant exponentials and replaces them with a unit or exponents $e^{\tau_{j}}$ of the first dimension according to the rule:

$$
\left(\left.e^{(m, \tau)}\right|_{m \in \Gamma_{0}}\right)^{\wedge}=e^{0}=1,\left(\left.e^{(m, \tau)}\right|_{m \in \Gamma_{j}}\right)^{\wedge}=e^{\tau_{j}}, j=\overline{1,3} .
$$


We now turn to the proof of the following statement.

Theorem 2. Suppose that conditions 1) and 2) are satisfied and the right-hand side

$$
\begin{aligned}
H(t, \tau)= & H_{0}(t)+\sum_{i=1}^{3} H_{i}(t) e^{\tau_{i}}+ \\
& +\sum_{2 \leq|m| \leq N_{H}}^{*} H^{m}(t) e^{(m, \tau)} \in U
\end{aligned}
$$

of the system (11) satisfies condition (12). Then the problem (11) under additional conditions

$$
<G(t, \tau), \chi_{k}(t) e^{\tau_{k}}>\equiv 0 \forall t \in\left[t_{0}, T\right], k=1,2,(20)
$$

where

$$
\begin{array}{r}
Q(t, \tau)=Q_{0}(t)+\sum_{k=1}^{3} Q_{k}(t) e^{\tau_{k}}+ \\
+\sum_{2 \leq|m| \leq N_{Q}}^{*} Q^{m}(t) e^{(m, \tau)}
\end{array}
$$

is the well-known vector function of the space $U$, is uniquely solvable in $U$.

Proof. Since the right-hand side of system (11) satisfies condition (12), this system has a solution in the space $U$ in the form (17), where $\alpha_{k}(t) \in C^{\infty}\left(\left[t_{0}, T\right], \mathrm{C}^{1}\right), k=1,2 \quad$ are arbitrary functions so far. We obey (18) the initial condition $z\left(t_{0}, 0\right)=z^{*}$. We obtain $\sum_{k=1}^{2} \alpha_{k}\left(t_{0}\right) \varphi_{k}\left(t_{0}\right)=z_{*}$, where is indicated

$$
\begin{gathered}
z_{*}=z^{*}+A^{-1}\left(t_{0}\right) H_{0}\left(t_{0}\right)- \\
-\left[\lambda_{5}\left(t_{0}\right) I-A\left(t_{0}\right)\right]^{-1} H_{5}\left(t_{0}\right)- \\
-\frac{\left(H_{1}\left(t_{0}\right), \chi_{2}\left(t_{0}\right)\right)}{\lambda_{1}\left(t_{0}\right)-\lambda_{2}\left(t_{0}\right)} \varphi_{2}\left(t_{0}\right)- \\
-\frac{\left(H_{2}\left(t_{0}\right), \chi_{1}\left(t_{0}\right)\right)}{\lambda_{2}\left(t_{0}\right)-\lambda_{1}\left(t_{0}\right)} \varphi_{1}\left(t_{0}\right)-\sum_{2 \leq|m| \leq N_{z}}^{*} z^{m}\left(t_{0}\right) .
\end{gathered}
$$

Multiplying scalarly the equality $\sum_{k=1}^{2} \alpha_{k}\left(t_{0}\right) \varphi_{k}\left(t_{0}\right)=z_{*}$ by $\chi_{j}\left(t_{0}\right)$ and taking into account the biorthogonality of the systems $\left\{\varphi_{k}(t)\right\}$ and $\left\{\chi_{j}(t)\right\}$, we find the values $\alpha_{k}\left(t_{0}\right)=\left(z_{*}, \chi_{k}\left(t_{0}\right)\right), k=1,2$.

Now we subordinate the solution (17) to the orthogonality condition (20). We write in more detail the right-hand side $G(t, \tau)$ of system (19):

$$
\begin{gathered}
G(t, \tau) \equiv-\frac{\partial}{\partial t}\left[z_{0}(t)+\sum_{k=1}^{2} \alpha_{k}(t) \varphi_{k}(t) e^{\tau_{k}}+\right. \\
+h_{12}(t) \varphi_{2}(t) e^{\tau_{1}}+h_{21}(t) \varphi_{1}(t) e^{\tau_{2}}+
\end{gathered}
$$

$$
\begin{aligned}
& \left.+z_{3}(t) e^{\tau_{3}}+\sum_{2 \leq|m| \leq N_{H}}^{*} P^{m}(t) e^{(m, \tau)}\right]+ \\
& +\frac{g(t)}{2}\left(e^{\tau_{1}} \sigma_{1}+e^{\tau_{2}} \sigma_{2}\right) B(t) \times \\
& \quad \times\left[z_{0}(t)+\sum_{k=1}^{2} \alpha_{k}(t) \varphi_{k}(t) e^{\tau_{k}}+\right. \\
& +h_{12}(t) \varphi_{2}(t) e^{\tau_{1}}+h_{21}(t) \varphi_{1}(t) e^{\tau_{2}}+ \\
& \left.+z_{3}(t) e^{\tau_{3}}+\sum_{2 \leq|m| \leq N_{H}}^{*} P^{m}(t) e^{(m, \tau)}\right]+ \\
& +R_{1}\left[z_{0}(t)+\sum_{k=1}^{2} \alpha_{k}(t) \varphi_{k}(t) e^{\tau_{k}}+\right. \\
& +h_{12}(t) \varphi_{2}(t) e^{\tau_{1}}+h_{21}(t) \varphi_{1}(t) e^{\tau_{2}}+ \\
& \left.+z_{3}(t) e^{\tau_{3}}+\sum_{2 \leq|m| \leq N_{H}}^{*} P^{m}(t) e^{(m, \tau)}\right]+Q(t, \tau) .
\end{aligned}
$$

Putting this function into the space $U$, we will have

$$
\begin{aligned}
& \hat{G}(t, \tau) \equiv-\frac{\partial}{\partial t}\left[z_{0}(t)+\sum_{k=1}^{2} \alpha_{k}(t) \varphi_{k}(t) e^{\tau_{k}}+\right. \\
& +h_{12}(t) \varphi_{2}(t) e^{\tau_{1}}+h_{21}(t) \varphi_{1}(t) e^{\tau_{2}}+ \\
& \left.+z_{3}(t) e^{\tau_{3}}+\sum_{2 \leq|m| \leq N_{H}}^{*} P^{m}(t) e^{(m, \tau)}\right]+ \\
& +\left\{\frac { g ( t ) } { 2 } ( e ^ { \tau _ { 1 } } \sigma _ { 1 } + e ^ { \tau _ { 2 } } \sigma _ { 2 } ) B ( t ) \left(z_{0}(t)+\right.\right. \\
& +\sum_{k=1}^{2} \alpha_{k}(t) \varphi_{k}(t) e^{\tau_{k}}+h_{12}(t) \varphi_{2}(t) e^{\tau_{1}}+ \\
& \left.+h_{21}(t) \varphi_{1}(t) e^{\tau_{2}}+z_{3}(t) e^{\tau_{3}}+\sum_{2 \leq|m| \leq N_{H}}^{*} P^{m}(t) e^{(m, \tau)}\right\}^{\wedge} \\
& +R_{1}\left[z_{0}(t)+\sum_{k=1}^{2} \alpha_{k}(t) \varphi_{k}(t) e^{\tau_{k}}+h_{12}(t) \varphi_{2}(t) e^{\tau_{1}}+\right. \\
& +h_{21}(t) \varphi_{1}(t) e^{\tau_{2}}+z_{3}(t) e^{\tau_{3}}+ \\
& \left.+\sum_{2 \leq|m| \leq N_{H}}^{*} P^{m}(t) e^{(m, \tau)}\right]+Q(t, \tau)= \\
& =-\frac{\partial}{\partial t}\left[z_{0}(t)+\sum_{k=1}^{2} \alpha_{k}(t) \varphi_{k}(t) e^{\tau_{k}}+\right. \\
& +h_{12}(t) \varphi_{2}(t) e^{\tau_{1}}+h_{21}(t) \varphi_{1}(t) e^{\tau_{2}}+ \\
& \left.+z_{3}(t) e^{\tau_{3}}+\sum_{2 \leq|m| \leq N_{H}}^{*} P^{m}(t) e^{(m, \tau)}\right]+ \\
& +\left\{\frac { g ( t ) } { 2 } B ( t ) \left(\mathrm{e}^{2 \tau_{1}} \sigma_{1} \alpha_{1}(t) \varphi_{1}(t)+\right.\right. \\
& +\mathrm{e}^{2 \tau_{1}} \sigma_{1} h_{12}(t) \varphi_{2}(t)+ \\
& +\mathrm{e}^{2 \tau_{2}} \sigma_{2} h_{21}(t) \varphi_{1}(t)+\mathrm{e}^{2 \tau_{2}} \sigma_{2} \alpha_{2}(t) \varphi_{2}(t)+
\end{aligned}
$$




$$
\begin{gathered}
+\mathrm{e}^{\tau_{1}+\tau_{2}} \sigma_{2} \alpha_{1}(t) \varphi_{1}(t)+ \\
+\frac{\mathrm{e}^{\tau_{1}+\tau_{2}} \sigma_{1} h_{21}(t) \varphi_{1}(t)}{\mathrm{e}^{\tau_{1}+\tau_{2}} \sigma_{1}} \alpha_{2}(t) \varphi_{2}(t) \\
+\frac{\mathrm{e}^{\tau_{1}+\tau_{2}} \sigma_{2} h_{12}(t) \varphi_{2}(t)}{\mathrm{e}^{\tau_{1}+\tau_{3}} \sigma_{1} z_{3}(t)+} \\
\left.+\mathrm{e}^{\tau_{2}+\tau_{3}} \sigma_{2} z_{3}(t)+\mathrm{e}^{\tau_{1}} \sigma_{1} z_{0}(t)+\mathrm{e}^{\tau_{2}} \sigma_{2} z_{0}(t)\right)+ \\
\left.+\frac{1}{2} g(t) B(t) \sum_{2 \leq|m| \leq N_{H}}^{*} P(t)^{m}\left(e^{m \tau+\tau_{3}} \sigma_{1}+e^{m \tau+\tau_{4}} \sigma_{2}\right)\right\}^{\wedge}+ \\
+R_{1}\left[z_{0}(t)+\sum_{k=1}^{2} \alpha_{k}(t) \varphi_{k}(t) e^{\tau_{k}}+h_{12}(t) \varphi_{2}(t) e^{\tau_{1}}+\right. \\
+h_{21}(t) \varphi_{1}(t) e^{\tau_{2}}+z_{3}(t) e^{\tau_{3}}+ \\
\left.+\sum_{2 \leq|m| \leq N_{H}}^{*} P^{m}(t) e^{(m, \tau)}\right]+Q(t, \tau) .
\end{gathered}
$$

The embedding operation acts only on resonant exponentials, leaving the coefficients unchanged at these exponents. Given that the expression

$$
\begin{aligned}
& R_{1}\left[z_{0}(t)+\sum_{k=1}^{2} \alpha_{k}(t) \varphi_{k}(t) e^{\tau_{k}}+h_{12}(t) \varphi_{2}(t) e^{\tau_{1}}+\right. \\
& \left.+h_{21}(t) \varphi_{1}(t) e^{\tau_{2}}+z_{3}(t) e^{\tau_{3}}+\sum_{2 \leq|m| \leq N_{H}}^{*} P^{m}(t) e^{(m, \tau)}\right]
\end{aligned}
$$

linearly depends on $\alpha_{1}(t)$ and $\alpha_{2}(t)$ (see the formula $\left(6_{1}\right)$, we conclude that, after the embedding operation, the function $\hat{G}(t, \tau)$ will linearly depend on scalar functions $\alpha_{1}(t)$ and $\alpha_{2}(t)$. Taking into account that under conditions (20), scalar multiplication by vector functions $\quad \chi_{k}(t) e^{\tau_{k}}, \quad$ containing only exponentials $e^{\tau_{k}}, k=1,2$, it is necessary to keep in the expression $G(t, \tau)$ only terms with exponents $e^{\tau_{1}}$ and $e^{\tau_{2}}$. Then condition (20) takes the form

$$
\begin{aligned}
<- & \frac{\partial}{\partial t}\left(\sum_{k=1}^{2} \alpha_{k}(t) \varphi_{k}(t) e^{\tau_{k}}+h_{12}(t) \varphi_{2}(t) e^{\tau_{1}}+\right. \\
& \left.+h_{21}(t) \varphi_{1}(t) e^{\tau_{2}}\right)+ \\
& +\left(\sum_{\left|m^{1}\right|=2: m^{1} \in \Gamma_{1}}^{N} w^{m^{1}}\left(\alpha_{1}(t), \alpha_{1}(t), t\right)\right) e^{\tau_{1}}+ \\
& +\left(\sum_{\left|m^{2}\right|=2: m^{2} \in \Gamma_{2}}^{N} w^{m^{2}}\left(\alpha_{1}(t), \alpha_{1}(t), t\right)\right) e^{\tau_{2}}+
\end{aligned}
$$

$$
\begin{array}{r}
+Q_{1}(t) e^{\tau_{1}}+Q_{2}(t) e^{\tau_{2}}, \chi_{k}(t) e^{\tau_{k}}>\equiv 0, \\
\forall t \in\left[t_{0}, T\right], k=1,2,
\end{array}
$$

where the functions $w^{m^{j}}\left(\alpha_{1}(t), \alpha_{2}(t), t\right), j=1,2$, depend on $\alpha_{1}(t)$ and $\alpha_{2}(t)$ in a linear way (see in $(* *)$ the underlined terms in and the formula $\left.\left(6_{1}\right)\right)$. Performing scalar multiplication here, we obtain linear ordinary differential equations with respect to the functions $\alpha_{k}(t), k=1,2$, involved in the solution (18) of system (11). Attaching the initial conditions $\alpha_{k}\left(t_{0}\right)=\left(z_{*}, \chi_{k}\left(t_{0}\right)\right), k=1,2$, calculated earlier to them, we find uniquely functions $\alpha_{k}(t)$, and, therefore, construct a solution (17) of the problem (19) in the space $U$ in a unique way. The theorem is proved.

As mentioned above, the right-hand sides of iterative problems $\left(\overline{10}_{k}\right)$ (with their consistent solution) may not belong to the space $U$. Then, according to [4] (p. 234), the righthand sides of these problems must be embedded into $U$, according to the above rule. As a result, we obtain the following problems:

$$
\begin{gathered}
L z_{0}(t, \tau) \equiv \sum_{j=1}^{3} \lambda_{j}(t) \frac{\partial z_{0}}{\partial \tau_{j}}-A(t) z_{0}-R_{0} z_{0}= \\
=h(t), z_{0}\left(t_{0}, 0\right)=z^{0} \\
L z_{1}(t, \tau)=\left[\frac{g(t)}{2}\left(e^{\tau_{1}} \sigma_{1}+e^{\tau_{2}} \sigma_{2}\right) B(t) z_{0}\right]^{\wedge}+ \\
\quad+R_{1} z_{0}-\frac{\partial z_{0}}{\partial t}, z_{1}\left(t_{0}, 0\right)=0 \\
L z_{2}(t, \tau)=\left[\frac{g(t)}{2}\left(e^{\tau_{1}} \sigma_{1}+e^{\tau_{2}} \sigma_{2}\right) B(t) z_{1}\right]^{\wedge}+ \\
+R_{1} z_{1}+R_{2} z_{0}-\frac{\partial z_{1}}{\partial t}, z_{0}\left(t_{0}, 0\right)=0 \\
\quad \ldots \\
L z_{k}(t, \tau)=\left[\frac{g(t)}{2}\left(e^{\tau_{1}} \sigma_{1}+e^{\tau_{2}} \sigma_{2}\right) B(t) z_{k-1}\right]^{\wedge}+ \\
+R_{k} z_{0}+\ldots+R_{1} z_{k-1}-\frac{\partial z_{k-1}}{\partial t}, z_{k}\left(t_{0}, 0\right)=0, k \geq 1
\end{gathered}
$$

(images of linear operators $\frac{\partial}{\partial t}$ and $R_{v}$ do not need to be embedded in space $U$, since these operators act from $U$ to $U)$. Such a replacement will not affect the construction of 
an asymptotic solution to the original problem (1) (or its equivalent problem (2)), so on the narrowing $\tau=\frac{\psi(t)}{\varepsilon}$ the series of problems $\left(\overline{10}_{k}\right)$ will coincide with the series of problems $\left(\overline{10}_{k}\right)$ (see [4], pp. 234-235].

Applying Theorems 1 and 2 to iterative problems $\left(\overline{10}_{k}\right)$, we find their solutions uniquely in space $U$ and construct series (7). As in [4], we prove the following statement.

Theorem 3. Let conditions 1) -2) be satisfied for system (2). Then, for $\varepsilon \in\left(0, \varepsilon_{0}\right]\left(\varepsilon_{0}>0\right.$ is sufficiently small) system (2) has a unique solution $z(t, \varepsilon) \in C^{1}\left([0, T], \mathrm{C}^{2}\right)$; at the same time there is an estimate

$$
\left\|z(t, \varepsilon)-z_{\varepsilon N}(t)\right\|_{C[0, T]} \leq c_{N} \varepsilon^{N+1}, n=0,1,2, \ldots,
$$

where $z_{\varepsilon N}(t)$ is the restriction on $\tau=\frac{\psi(t)}{\varepsilon}$ of the $N$-th partial sum of the series (7) (with coefficients $z_{k}(t, \tau) \in U$, satisfying the iterative problems $\left.\left(\overline{10}_{k}\right)\right)$ and the constant $c_{N}>0$ does not depend on $\varepsilon$ at $\varepsilon \in\left(0, \varepsilon_{0}\right]$.

\section{Construction of a solution to the first iterative problem}

Using Theorem 1, we try to find a solution to the first iterative problem $\left(\overline{10}_{0}\right)$. Since the righthand side $h(t)$ of the system $\left(\overline{10}_{0}\right)$ satisfies condition (12), this system (according to (17)) has a solution in the space $U$ in the form

$$
z_{0}(t, \tau)=z_{0}^{(0)}(t)+\sum_{k=1}^{2} \alpha_{k}^{(0)}(t) \varphi_{k}(t) e^{\tau_{k}},
$$

where $\quad \alpha_{k}^{(0)}(t) \in C^{\infty}\left(\left[t_{0}, T\right], C^{1}\right)$ are $\quad$ arbitrary functions, $\quad k=1,2, z_{0}^{(0)}(t)=-A^{-1}(t) h(t)$. Subordinating $(21)$ to the initial condition $z_{0}\left(t_{0}, 0\right)=z^{0}$, we have

$$
\begin{gathered}
z_{0}^{(0)}\left(t_{0}\right)+\sum_{k=1}^{2} \alpha_{k}^{(0)}\left(t_{0}\right) \varphi_{k}\left(t_{0}\right)=z^{0} \Leftrightarrow \\
\Leftrightarrow \sum_{k=1}^{2} \alpha_{k}^{(0)}\left(t_{0}\right) \varphi_{k}\left(t_{0}\right)=z^{0}+A^{-1}\left(t_{0}\right) h\left(t_{0}\right) .
\end{gathered}
$$

Multiplying this equality scalarly $\chi_{j}\left(t_{0}\right)$ and taking into account the biorthogonality of the systems $\left\{\varphi_{k}(t)\right\}$ and $\left\{\chi_{j}(t)\right\}$, we find the values

$$
\alpha_{k}^{(0)}\left(t_{0}\right)=\frac{1}{2}\left(z^{0}+A^{-1}\left(t_{0}\right) h\left(t_{0}\right), \chi_{k}\left(t_{0}\right)\right), k=1,2 .
$$

For a complete calculation of the functions $\alpha_{k}^{(0)}(t)$, we proceed to the next iterative problem $\left(\overline{10}_{1}\right)$. Substituting the solution (21) of the system $\left(\overline{10}_{0}\right)$ into it, we arrive at the following system:

$$
\begin{gathered}
L z_{1}(t, \tau)=-\frac{d}{d t} z_{0}^{(0)}(t)-\sum_{k=1}^{2} \frac{d}{d t}\left(\alpha_{k}^{(0)}(t) \varphi_{k}(t)\right) e^{\tau_{k}}+ \\
+\frac{K(t, t) z_{0}^{(0)}(t)}{\lambda_{3}(t)} e^{\tau_{3}}-\frac{K\left(t, t_{0}\right) z_{0}^{(0)}\left(t_{0}\right)}{\lambda_{3}\left(t_{0}\right)}+ \\
+\left[\frac{g(t)}{2}\left(e^{\tau_{1}} \sigma_{1}+e^{\tau_{2}} \sigma_{2}\right) B(t) \times\right. \\
\left.\times\left(z_{0}^{(0)}(t)+\sum_{k=1}^{2} \alpha_{k}^{(0)}(t) \varphi_{k}(t) e^{\tau_{k}}\right)\right]^{\wedge}+ \\
+\sum_{j=1}^{2}\left\lfloor\frac{\left(K(t, t) \alpha_{j}^{(0)}(t) \varphi_{j}(t)\right)}{\lambda_{j}(t)} e^{\tau_{j}}-\right. \\
\left.-\frac{\left(K\left(t, t_{0}\right) \alpha_{j}^{(0)}\left(t_{0}\right) \varphi_{j}\left(t_{0}\right)\right)}{\lambda_{j}\left(t_{0}\right)}\right],
\end{gathered}
$$

here we used the expression $\left(6_{1}\right)$ for $R_{1} z(t, \tau)$ and took into account that when $z(t, \tau)=z_{0}(t, \tau)$ in the sum $\left(6_{1}\right)$ only terms with $e^{\tau_{1}}, e^{\tau_{2}}$ and $e^{\tau_{5}}$ remain). We calculate

$$
\begin{gathered}
M=\left[\frac{g(t)}{2}\left(e^{\tau_{1}} \sigma_{1}+e^{\tau_{2}} \sigma_{2}\right) B(t) \times\right. \\
\left.\times\left(z_{0}^{(0)}(t)+\sum_{k=1}^{2} \alpha_{k}^{(0)}(t) \varphi_{k}(t) e^{\tau_{k}}\right)\right]^{\wedge}= \\
=\frac{1}{2} g(t) B(t)\left[e^{\tau_{1}} \sigma_{1} z_{0}(t)+\mathrm{e}^{2 \tau_{1}} \sigma_{1} \alpha_{1}(t) \varphi_{1}(t)+\right. \\
+\mathrm{e}^{\tau_{1}+\tau_{2}} \sigma_{1} \alpha_{2}(t) \varphi_{2}(t)+\mathrm{e}^{\tau_{2}} \sigma_{2} z_{0}(t)+ \\
\left.+\mathrm{e}^{\tau_{1}+\tau_{2}} \sigma_{2} \alpha_{1}(t) \varphi_{1}(t)+\mathrm{e}^{2 \tau_{2}} \sigma_{2} \alpha_{2}(t) \varphi_{2}(t)\right]^{\wedge}= \\
=\frac{1}{2} g(t) B(t)\left[e^{\tau_{1}} \sigma_{1} z_{0}(t)+\mathrm{e}^{2 \tau_{1}} \sigma_{1} \alpha_{1}(t) \varphi_{1}(t)+\right. \\
+\sigma_{1} \alpha_{2}(t) \varphi_{2}(t)+\mathrm{e}^{\tau_{2}} \sigma_{2} z_{0}(t)+ \\
\left.+\sigma_{2} \alpha_{1}(t) \varphi_{1}(t)+\mathrm{e}^{2 \tau_{2}} \sigma_{2} \alpha_{2}(t) \varphi_{2}(t)\right] .
\end{gathered}
$$


Since $e^{\tau_{1}+\tau_{2}}=1$, then system (23) after embedding takes the form

$$
\begin{gathered}
L z_{1}(t, \tau)=-\frac{d}{d t} z_{0}^{(0)}(t)-\sum_{k=1}^{2} \frac{d}{d t}\left(\alpha_{k}^{(0)}(t) \varphi_{k}(t)\right) e^{\tau_{k}}+ \\
+\frac{K(t, t) z_{0}^{(0)}(t)}{\lambda_{3}(t)} e^{\tau_{3}}-\frac{K\left(t, t_{0}\right) z_{0}^{(0)}\left(t_{0}\right)}{\lambda_{3}\left(t_{0}\right)}+ \\
+\frac{1}{2} g(t) B(t)\left[e^{\tau_{1}} \sigma_{1} z_{0}(t)+e^{2 \tau_{1}} \sigma_{1} \alpha_{1}(t) \varphi_{1}(t)+\right. \\
+\sigma_{1} \alpha_{2}(t) \varphi_{2}(t)+e^{\tau_{2}} \sigma_{2} z_{0}(t)+ \\
\left.+\sigma_{2} \alpha_{1}(t) \varphi_{1}(t)+e^{2 \tau_{2}} \sigma_{2} \alpha_{2}(t) \varphi_{2}(t)\right]+ \\
+\sum_{j=1}^{2}\left\lfloor\frac{\left(K(t, t) \alpha_{j}^{(0)}(t) \varphi_{j}(t)\right)}{\lambda_{j}(t)} e^{\tau_{j}}-\right. \\
\left.-\frac{\left(K\left(t, t_{0}\right) \alpha_{j}^{(0)}\left(t_{0}\right) \varphi_{j}\left(t_{0}\right)\right)}{\lambda_{j}\left(t_{0}\right)}\right\rfloor,
\end{gathered}
$$

This system is solvable in the space $U$ if and only if the conditions of orthogonality are satisfied:

$$
\begin{gathered}
\left\langle-\sum_{k=1}^{2} \frac{d}{d t}\left(\alpha_{k}^{(0)}(t) \varphi_{k}(t)\right) e^{\tau_{k}}+\frac{1}{2} g(t) B(t)\left[e^{\tau_{1}} \sigma_{1} z_{0}(t)+\right.\right. \\
\quad+e^{2 \tau_{1}} \sigma_{1} \alpha_{1}(t) \varphi_{1}(t)+\sigma_{1} \alpha_{2}(t) \varphi_{2}(t)+ \\
\left.+e^{\tau_{2}} \sigma_{2} z_{0}(t)+\sigma_{2} \alpha_{1}(t) \varphi_{1}(t)+e^{2 \tau_{2}} \sigma_{2} \alpha_{2}(t) \varphi_{2}(t)\right]+ \\
\left.+\sum_{i=1}^{2} \frac{\left(K(t, t) \alpha_{i}^{(0)}(t) \varphi_{i}(t)\right)}{\lambda_{i}(t)} e^{\tau_{i}}, \chi_{j}(t) e^{\tau_{j}}\right\rangle \equiv 0, j=1,2 .
\end{gathered}
$$

Performing scalar multiplication here, we obtain an inhomogeneous system of decomposing differential equations:

$$
\begin{gathered}
-\frac{d \alpha_{1}^{(0)}(t)}{d t}-\left(\dot{\varphi}_{1}(t), \chi_{1}(t)\right) \alpha_{1}^{(0)}(t)+ \\
+\frac{\left(K(t, t), \chi_{1}(t)\right)}{\lambda_{1}(t)} \alpha_{1}^{(0)}(t)+ \\
+\frac{1}{2} g(t) \sigma_{1}\left(B(t) z_{0}(t), \chi_{1}(t)\right) \equiv 0 \\
-\frac{d \alpha_{2}^{(0)}(t)}{d t}-\left(\dot{\varphi}_{2}(t), \chi_{2}(t)\right) \alpha_{2}^{(0)}(t)+ \\
-\frac{d \alpha_{2}^{(0)}(t)}{d t}-\left(\dot{\varphi}_{2}(t), \chi_{2}(t)\right) \alpha_{2}^{(0)}(t)+ \\
+\frac{1}{2} g(t) \sigma_{2}\left(B(t) z_{0}(t), \chi_{2}(t)\right) \equiv 0 .
\end{gathered}
$$

Adding the initial conditions (22) to this system, we find uniquely functions $\alpha_{k}^{(0)}(t)$, $k=1,2$, and, therefore, uniquely calculate the solution $(21)$ of the problem $\left(\overline{10}_{0}\right)$ in the space $U$. Moreover, the main term of the asymptotic behavior of the solution to problem (2) has the form

$$
z_{\varepsilon 0}(t)=z_{0}^{(0)}(t)+\sum_{k=1}^{2} \alpha_{k}^{(0)}(t) \varphi_{k}(t) e^{\frac{1}{\varepsilon} \int_{t_{0}}^{t} \lambda_{k}(\theta) d \theta},
$$

where the functions $\alpha_{k}^{(0)}\left(t_{0}\right)$ satisfy problem (22), (24), $z_{0}^{(0)}(t)=-A^{-1}(t) h(t)$.

This work was supported by Grant No. AP05133858 of the Science Committee of the Ministry of Education and Science of the Republic of Kazakhstan.

\section{References:}

[1]. Daletsky, Y.L., The asymptotic method for some differential equations with oscillating coefficients, DAN USSR, Vol. 143, 1962, 1026--1029. (in Russian)

[2]. Feschenko, S.F., Shkil, N.I., Nikolenko, L.D. Asymptotic methods in the theory of linear differential equations, Kiev, Naukova Dumka, 1966. (in Russian)

[3]. Shkil, N.I., Asymptotic methods in differential equations, Kiev, Naukova Dumka, 1971. (in Russian)

[4]. Lomov, S.A., Introduction to General Theory of Singular Perturbations, vol. 112 of Translations of Math. Monographs, American Math. Society, Providence, USA, 1992.

[5]. Ryzhih, A.D. Asymptotic solution of a linear differential equation with a rapidly oscillating coefficient, Trudy MEI, Vol. 357, 1978, 92--94. (in Russian)

[6]. Kalimbetov, B.T., Safonov, V.F., A regularization method for systems with a nonstable spectral value for the kernel of the integral operator, J. Differ. Equations, Vol. 31. No 4, 1995, pp. 647-656.

[7]. Safonov, V.F., Tuichiev, O.D., Regularization of singularly perturbed integral equations with rapidly varying kernels and their asymptotics, J. Differ. Equations, Vol. 33. No 9, 1997, pp. 12031215.

[8]. Bobodzhanov, A.A., Safonov, V.F., Volterra integral equations with rapidly 
varying kernels and their asymptotic integration, Sibir. Math. J., Vol. 192. No 8, 2001, pp. 1139-1164.

[9]. Kalimbetov, B.T., Temirbekov, M.A., Khabibullaev, Zh.O., Asymptotic solution of singular perturbed problems with an instable spectrum of the limiting operator, Abstract and Applied Analysis. Vol. 2012, Article ID 120192.

[10].Bobodzhanov, A.A., Safonov, V.F., Regularized Asymptotic Solutions of Singularly Perturbed Integral Systems with a Diagonal Degeneration of the Kernel, J. Differ. Equations, Vol. 37. No 10, 2001, pp. 1399-1411.

[11].Bobodzhanov, A.A., Safonov, V.F., Asymptotic analysis of integro-differential systems with an unstable spectral value of the integral operator's kernel, J. Comput. Math. Math. Phys., Vol. 47. No 1. 2007, pp. 65-79.

[12].Kalimbetov, B.T., Imanbaev, N.S., Tashimov, L., Khabibullaev, Zh.O., Regularized asymptotical solutions of integro-differential systems with spectral singularities, Advances in Difference Equations, 2013, 2013:109 doi:10.1186/1687-1847-2013-109.

[13].Bobodzhanov, A.A., Safonov, V.F., Regularized asymptotics of solutions to integro-differential partial differential equations with rapidly varying kernels, Ufa Math. J., Vol. 10, No 2, 2018, pp. 3-13.

[14].Bobodzhanov, A.A., Safonov, V.F., $A$ generalization of the regularization method to the singularly perturbed integrodifferential equations with partial derivatives, Russian Math., Vol. 62. No 3, 2018, pp. 6-17.
[15].Kalimbetov, B.T., Pardaeva, N.A., Sharipova, L.D., Asymptotic solutions of integro-differential equations with partial derivatives and with rapidly varying kernel, SEMR, Vol. 16. No 113, 2019, pp. 16231631. DOI 10.33048/semi.2019.16.113.

[16].

alimbetov, B.T., Safonov, V.F., Integrodifferentiated singularly perturbed equations with fast oscillating coefficients, Bulletin KSU-mathematics, Vol. 94. No 2, 2019, pp. 33-47. DOI $10.31489 / 2019 \mathrm{M} 2 / 33-47$.

[17].Kalimbetov, B.T., Temirbekov, A.N., Tolep, A.S., Asymptotic solutions of scalar integro-differential equations with partial derivatives and with fast oscillating coefficients, EJPAM, Vol. 13. No 2, 2020, pp. 287-302.

[18].Bobodzhanov, A.A., Kalimbetov, B.T., Safonov, V.F., Integro-differential problem about parametric amplification and its asymptotical integration, IJAM, Vol. 33 No. 2 2020, 331-353 doi: http://dx.doi.org/10.12732/ijam.v3312.12

[19].Safonov, V.F., Bobodzhanov, A.A., Course of higher mathematics. Singularly perturbed equations and the regularization method: textbook, Moscow, Publishing House of MPEI, 2012. (in Russian)

\section{Creative Commons Attribution License 4.0 (Attribution 4.0 International, CC BY 4.0)}

This article is published under the terms of the Creative Commons Attribution License 4.0 https://creativecommons.org/licenses/by/4.0/deed.en_US 